\title{
Inhibition of Intracellular Survival of Multi Drug Resistant Clinical Isolates of Mycobacterium tuberculosis in Macrophages by Curcumin
}

\author{
Pramod Kumar Gupta, Savita Kulkarni* and Ramakrishna Rajan \\ Radiation Medicine Centre, Bio-Medical Group, Bhabha Atomic Research Centre, TMH Annexe, Parel, Mumbai \\ 400012, India
}

\begin{abstract}
Curcuma longa commonly known as turmeric has been used in Indian Ayurvedic medicine as a constituent to treat various disorders. It is by now clear that principal curcuminoid of turmeric; curcumin, a yellow pigment, is responsible for these beneficiary activities. The aim of the present study was to evaluate anti-mycobacterial effect of curcumin $(\mathrm{CMN})$ on intracellular growth of MDR clinical isolates of Mycobacterium tuberculosis (MTB). Curcumin was evaluated for its efficacy to inhibit the intracellular growth of MTB H37Rv and two MDR clinical isolates in Raw 264.7 cell line using CFU assay. Resazurin microtiter plate assay (REMA) was used to evaluate its direct anti-mycobacterial activity.

Curcumin, though did not show direct anti-mycobacterial activity against three MTB strains, exhibited dose dependent inhibition of intracellular growth for MTB H37Rv as well as two MDR clinical isolates. These results suggest that CMN could be a potential candidate for future, novel adjunctive anti-TB therapy.
\end{abstract}

Keywords: Anti-mycobacterial activity, Curcumin, MDR, Tuberculosis.

\section{INTRODUCTION}

Tuberculosis (TB) caused by Mycobacterium tuberculosis (MTB), is a public-health problem worldwide with a global mortality of 1.4 million with 350,000 deaths each year in India $[1,2]$. The situation is further exacerbated with increasing incidence of multi-drug resistant (MDR), extensively drug resistant (XDR) TB and co-infection of HIV.

Current drug regimens are far from effectively controling the incidence of drug resistance of MTB. Hence, the discovery of novel agents targeting host rather than pathogen is need of hour [3]. Since, MTB is known to alter signaling required for the production of immunostimulatory cytokines and effectors molecules in the host [4], immunomodulation beneficial to host, appears a viable strategy to control the pathogen.

Curcumin (CMN- [diferuloylmethane or 1,7-bis (4hydroxy-3-methoxy-phenyl) hepta-1, 6-diene-3, 5-dione)]) is a yellow pigment from the rhizomes of perennial herb Curcuma longa commonly known as turmeric. It has been shown to modulate various molecular targets in malignancy and signaling cascades involved in both innate and acquired immune response and, hence is utilized to treat various disorders including arthritis, cardiovascular disorders, cancers, and other pathologies [5]. In addition, CMN and largely its analogs have exhibited the antimicrobial and anti-mycobacterial activity in nonpathogenic species [6].

\footnotetext{
*Address correspondence to this author at the Radiation Medicine Centre, Bio-Medical Group, Bhabha Atomic Research Centre, TMH Annexe, Parel, Mumbai 400012, India; Cel: +91-22-24134960; Tel: +91-9821083165;

Ext: 211; Fax: +91-22-24157098; E-mail: savita.kulkarni1@gmail.com
}

The aim of the present study was to evaluate antimycobacterial efficiency of CMN on intracellular growth of MDR clinical isolates of MTB inside RAW 264.7 cells at lower doses.

\section{MATERIALS\& METHODS}

\subsection{Reagents}

Curcumin, resazurin sodium salt and MTT were obtained from Sigma Aldrich. Cell culture media DMEM and fetal calf serum were purchased from Gibco, Life Technologies. Bacterial culture media Middlebrook 7H9, 7H11, ADC and OADC were purchased from Difco, BD biosciences. LJ tubes containing antibiotics were purchased from EOS labs, Mumbai.

\subsection{Bacterial Cultures and Cell Lines}

M. tuberculosis clinical isolates strain-1 and strain-2 were recovered from patients in Tata memorial hospital, and KEM hospital, Mumbai (INDIA). The laboratory strain $\mathrm{H} 37 \mathrm{Rv}$ was also included in the study. All M. tuberculosis strains were grown in Middlebrook 7H9 medium supplemented with ADC (albumin-dextrose complex), and containing $0.05 \%$ Tween 80 for 10 days $37^{\circ} \mathrm{C}$ with daily agitation; working stocks were prepared $\left(10^{8}\right.$ bacilli $\left./ \mathrm{ml}\right)$ stored at $-70^{\circ} \mathrm{C}$ until use. All procedures were carried out in a Biosafety Level III (BSL III) laboratory. Murine macrophage cell line RAW 264.7 was obtained from NCCS Pune, India and maintained in DMEM at $37^{\circ} \mathrm{C}$, in an atmosphere containing $5 \%$ $\mathrm{CO} 2$. 


\subsection{Drug Susceptibility Testing}

Drug susceptibility of two clinical isolates to rifampicin, ethambutol, streptomycin, isoniazide, pyrazinamide, kanamycin, ethionamide clarithromycin, ciprofloxacin, cycloserine, amikacin, p-amino salicylic acid, rifabutin, was performed by standard proportion method [7].

\subsection{Resazurin Microtiter Plate Assay (REMA)}

The risazurin plate assay was performed in Middlebrook 7H9 broth supplemented with ADC, as described [8]. Briefly, dilutions of CMN were prepared in $7 \mathrm{H} 9$ broth and $100 \mu \mathrm{l}$ of this was dispensed in sterile 96 well plates at concentrations of $5-50 \mu \mathrm{M}$. Isoniazid was used as positive control. One hundred microliters of inoculum containing $10^{4}$ MTB cells was added to each well. Periphery wells were filled with sterile water to minimize evaporation. The plate was covered, sealed with paraffin, and incubated a $37^{\circ} \mathrm{C}$ for 7 days. Thirty microliters of resazurin solution were added to each well, and the plate was incubated overnight. A change in color from blue to pink signified the growth of bacteria, and the lowest concentration of drug that prevented this change in color was defined as MIC.

\subsection{Cell viability Assay}

Effect of CMN treatment on viability of RAW 264.7 cell was measured by MTT (3-(4,5-dimethylthiazol-2-yl)-2,5diphenyl tetrazolium bromide) assay as described [9]. Briefly, $5 \times 10^{3}$ RAW264.7 cells were seeded per well, in 96 well plates, kept in incubator at $37^{\circ} \mathrm{C}$, with $5 \% \mathrm{CO}_{2}$ overnight. Following day $\mathrm{CMN}$ was added at concentration from $2 \mu \mathrm{M}$ to $100 \mu \mathrm{M}$ and plates were incubated further for another 24 hours. MTT was added and after 3 hours incubation at $37^{\circ} \mathrm{C}$, lysis buffer was added (10\% SDS, $25 \%$ dimethylformamide, $\mathrm{pH}$ 4.7). Twenty four hours later, absorbance was measured at $570 \mathrm{~nm}$ and $630 \mathrm{~nm}$ with a microtiter plate reader.

\section{Macrophage Infection and CFU Assay}

All MTB strains growing in Middlebrook 7H9 broth supplemented with ADC at mid-log growth phase were harvested. Single cell suspensions were prepared in DMEM medium after vortexing washed pellets with the glass beads, followed by repeated passage through $26 \mathrm{G}$ needle. RAW 264.7 cells were seeded at a density of $2 \times 10^{5}$ cells/well and infected with MTB strains at an MOI of 5, for 3 hours. Cells were washed with pre-warmed media followed by treatment with amikacin $(10 \mu \mathrm{g} / \mathrm{ml})$ to kill the extracellular bacteria [10]. CMN was added at various concentrations $(10 \mu \mathrm{M}, 25$ $\mu \mathrm{M} 50 \mu \mathrm{M})$ and cells were incubated for 48 and 96 hours. For CFU assay, infected cells were washed with PBS and lysed with $0.01 \%$ SDS containing PBS at different time points. Serial dilutions were prepared in PBS and plated on 7H11 medium supplemented with OADC.

\section{Statistical Analysis}

SigmaStat (version 3.5) was used for statistical analysis. Data were presented as mean \pm standard deviation $(\mathrm{P}<0.05)$.

\section{RESULTS \\ MDR Status of Strains}

Drug sensitivity testing of both the clinical isolates demonstrated the multidrug resistant status of both isolates. Strain-1 exhibited resistance to 6 drugs and strain-2 showed resistance to 10 drugs (Table 1)

\section{Cell Viability Assay}

Cytotoxicity of CMN on pathogen (MTB) and host cells (RAW 264.7) was evaluated independently by REMA and MTT assay respectively. REMA demonstrated the absence of direct anti-mycobacterial activity of CMN against MTB strains up to $50 \mu \mathrm{M}(18.41 \mu \mathrm{g} / \mathrm{ml})$. MTT assay showed that concentrations of $\mathrm{CMN}$ up to $100 \mu \mathrm{M}$ were non-toxic to RAW 264.7 cells (Fig. 1).

\section{Inhibition of Intracellular Survival of MTB Strains by Curcumin}

There was a dose dependent inhibition of intracellular survival of MTB strains, when MTB infected macrophages were treated with the various doses $(10 \mu \mathrm{M}, 25 \mu \mathrm{M} 50 \mu \mathrm{M})$ of CMN for 48 (Fig. 2, A) and 96 hours (Fig. 2, B). Table 2 illustrates the values of percent inhibition of intracellular survival of MTB inside macrophages.

Percentage of inhibition of intracellular survival of MTB $\mathrm{H} 37 \mathrm{Rv}$ after $\mathrm{CMN}$ treatment $(10 \mu \mathrm{M})$ was significantly higher than strain $-2(p<0.05)$ and strain $-1(\mathrm{p}<0.001)$ at 48 hours post treatment. There was no significant difference observed among the percentage inhibition with all three strains at two higher doses $(25 \mu \mathrm{M}, 50 \mu \mathrm{M})$ at the same time point. Similarly, percent inhibition of intracellular survival of H37Rv after CMN $(50 \mu \mathrm{M})$ treatment is significantly higher than strain $2(\mathrm{p}<0.001)$ and strain $1(\mathrm{p}<0.05)$ after 96 hours. No significant difference was observed at $10 \mu \mathrm{M}$ and $25 \mu \mathrm{M}$ CMN treatment at 96 hours.

\section{DISCUSSION}

The rapid emergence of MDR and XDR TB, which is difficult to treat, is an issue of great concern; hence, now time has come to think about some novel therapeutic ap-

Table 1. Drug Sensitivity of Two Clinical Isolates

\begin{tabular}{|l|l|l|}
\hline \multicolumn{1}{|c|}{ Strains } & \multicolumn{1}{|c|}{ Resistant } & Sensitive \\
\hline \hline Strain-1 & $\begin{array}{l}\text { Rifampicin, Ethambutol, Streptomycin, Pyrazinamide, Kanamycin, } \\
\text { Ethionamide }\end{array}$ & $\begin{array}{l}\text { Isoniazide, Clarithromycin, Ciprofloxacin, Cycloserine, } \\
\text { Amikacin, P-Amino salicylic acid, Rifabutin }\end{array}$ \\
\hline Strain-2 & $\begin{array}{l}\text { Ethambutol, Streptomycin, Isoniazide, Pyrazinzmide, Rifampicin, } \\
\text { cycloserin, Ethionamide, Kanamycin, Clarithromycin, Rifabutin }\end{array}$ & Ciprofloxacin, Amikacin, P-Amino salicylicacid, \\
\hline
\end{tabular}




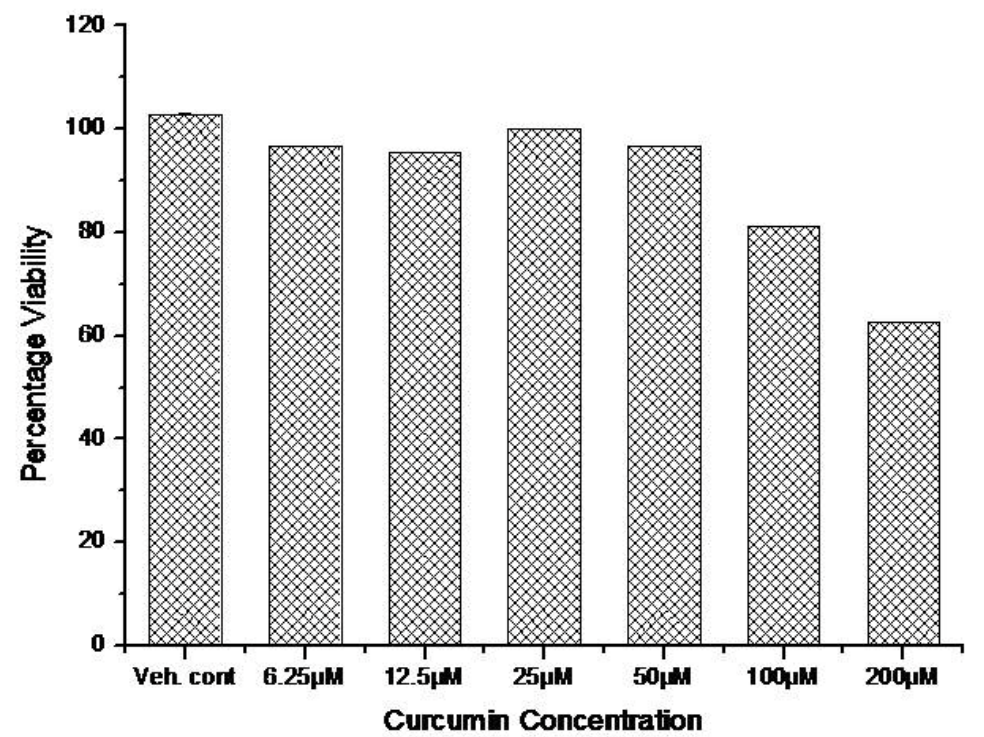

Fig. (1). Effect of curcumin treatment on viability of RAW 264.7 macrophages.

Figure represents the percent viability of RAW cells 24 hours after CMN treatment. CMN treatment did not exhibited cell cytotoxicity upto $100 \mu \mathrm{M}$ concentration. Data (Mean $\pm \mathrm{SD}$ ) shown are from a representative experiment of the three individual experiments.

Table 2. Percentage Inhibition of Intracellular Survival of MTB Strains After CMN Treatment

\begin{tabular}{|c|c|c|c|c|c|c|}
\hline \multirow{2}{*}{$\begin{array}{c}\text { CMN CONC } \\
(\mu \mathrm{M})\end{array}$} & \multicolumn{6}{|c|}{ Percentage inhibition \pm S.D. $(\mathbf{P}<\mathbf{0 . 0 5})$} \\
\hline & 48 hours & 96 hours & 48 hours & 96 hours & 48 hours & 96 hours \\
\hline 10 & $53.69 \pm 10.70$ & $36.25 \pm 5.7$ & $32.88 \pm 6.57$ & $26.95 \pm 2.14$ & $35.09 \pm 13.66$ & $29.15 \pm 3.84$ \\
\hline 25 & $57.74 \pm 7.48$ & $63.26 \pm 6.9$ & $47.95 \pm 6.54$ & $59.56 \pm 3.30$ & $46.32 \pm 10.53$ & $58.28 \pm 4.45$ \\
\hline Vehicle & $7.47 \pm 3.7$ & $3.98 \pm 1.97$ & $6.1 \pm 3.56$ & $1.51 \pm 1.53$ & $7.55 \pm 4.75$ & $2.65 \pm 2.27$ \\
\hline
\end{tabular}

proaches targeting the host immunity. It has been reported that use of TLR9 agonist (CpG oligonucleotides) partially inhibits the growth of intracellular MTB [11]. Further many immunomodulators are currently in clinical trial because of their anti-infective properties against various pathogens [12]. The aim of the present study was to evaluate the ability of CMN for the inhibition of intracellular survival of MTB in macrophages. Earlier reports demonstrated the direct antimycobacterial activity of CMN against non pathogenic MTB H37Ra at higher dose [6] (MIC: $100 \mu \mathrm{g} / \mathrm{ml}$ ), and nontuberculosis mycobacteria (e.g. M. kansasii, M simiae, M.smegmatis etc.) [13] but in the present study, lower dose $(18.14 \mu \mathrm{g} / \mathrm{ml})$ of CMN has been used which does not exhibit any direct anti-mycobacterial property. The percent inhibition observed for of $\mathrm{H} 37 \mathrm{Rv}$ was significantly higher than drug resistant strain- 1 and strain-2, at $10 \mu \mathrm{M}$ and $25 \mu \mathrm{M}$ of CMN dose after 48 hours. This may be attributed to i) highly drug resistant status of two clinical isolates, and ii) differential host response evoked by these highly virulent strains. The mode of action of inhibition is dependent on the host response than the direct action on MTB. Our group has already reported that both clinical isolates belong to two different genetic lineages and have displayed differential innate immune responses in human monocytic cell line THP-1 [14].
Interestingly percentage inhibition of intracellular survival of all the three strains at 96 hours of CMN treatment $(10 \mu \mathrm{M})$ was lower than the inhibition observed at 48 hours of CMN treatment. The degradation of CMN over the extended time period might have led to poor efficacy and thus decreased percent inhibition at this concentration. Though CMN did not exhibit any direct anti-mycobaterial effect up to $50 \mu \mathrm{M}$, the treatment of MTB infected macrophages with CMN resulted in dose dependent inhibition of intracellular growth of all the three strains under the study.

$\mathrm{CMN}$ is known to induce the expression of cathelicidin, an antimicrobial peptide in human monocytic cell line U-937 [15] and cathelicidin was independently shown to be involved in intracellular killing of MTB in murine macrophages [16]. We envisage that, the induction of antimicrobial peptides in MTB infected macrophages after CMN treatment may be a potential mode of action by which it inhibited the survival of MTB strains in macrophages.

Finally the absence of direct anti-mycobacterial effect of $\mathrm{CMN}$ indicates that inhibition of intracellular survival of MTB strains inside macrophages is probably the result of modulation of macrophage responses. This inhibition, unlike antibiotics, is achieved by targeting the host rather than the 

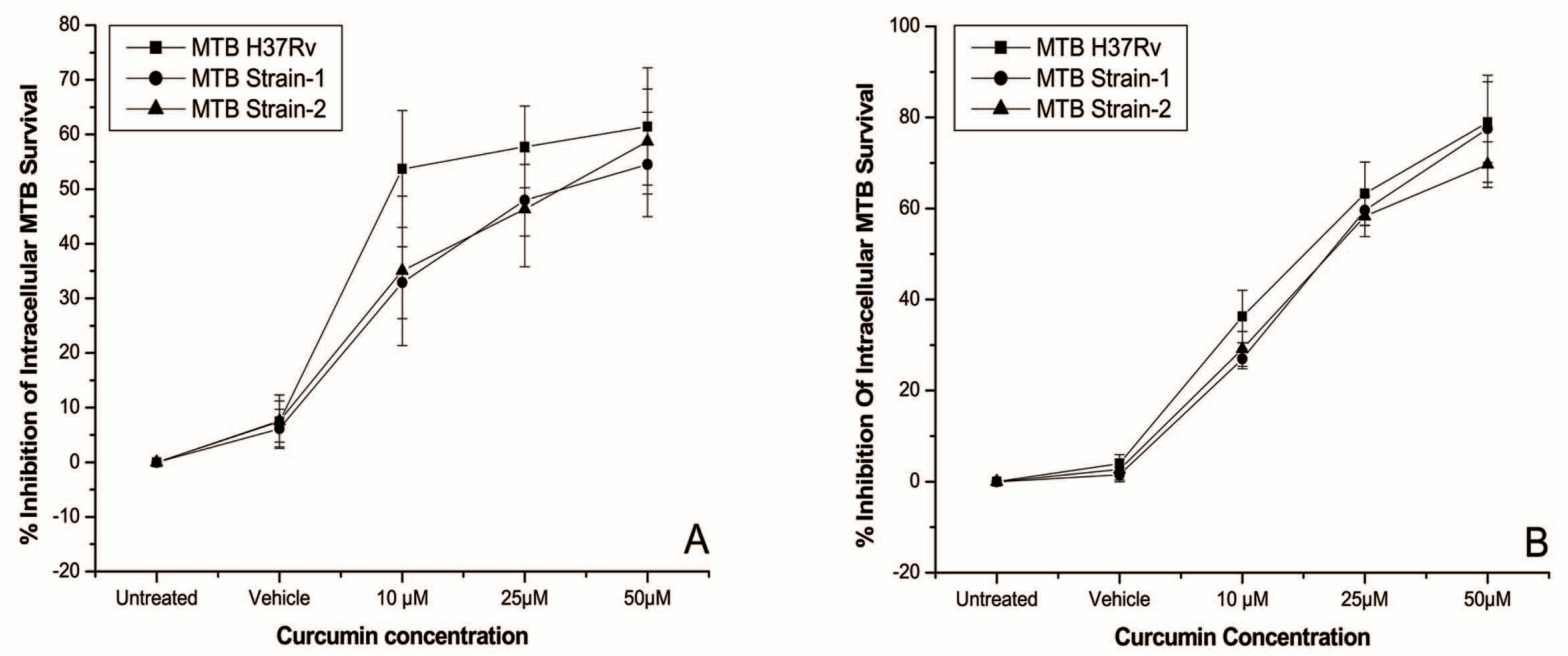

Fig. (2). Effect of curcumin treatment on intracellular survival of MDR clinical isolates in RAW 264.7 macrophages: Figure depicts the percentage inhibition of intracellular survival of MTB H37Rv and MDR clinical isolates in RAW 264.7 macrophages. Macrophages were infected with MTB H37Rv and two clinical isolates at MOI 5, followed by treatment of CMN $10 \mu \mathrm{M}, 25 \mu \mathrm{M}$, and $50 \mu \mathrm{M}$, and vehicle i.e. DMSO alone. Cells were lysed at 48 hours and 96 hours, plated on 7H11 Middlebrook plates for CFU enumeration. Number of colonies were counted after 21 days and expressed as percent inhibition of intracellular survival. For untreated cells percentage of inhibition was taken zero. A) - 48 hours Post treatment; B) - 96 hours post treatment. Percent inhibition of intracellular survival of all MTB strains increased significantly at both time points, and at all three concentrations of $\mathrm{CMN}$ in comparison to vehicle control, (P<0.001). One way ANOVA (Tukey's method) was used for statistical analysis. Data (Mean \pm SD) shown are from a representative experiment of the three individual experiments.

pathogen. This is a novel finding and similar reports on antimycobacterial effects of natural products are not available.

\section{CONCLUSION}

Rapid emergence of drug resistance in MTB strains even to newly discovered antibiotics is very alarming situation. Thus, in this era of MDR and XDR tuberculosis, conventional drug regimes are unable to control the pathogen and hence, in this scenario targeting the host rather than the pathogen to control tuberculosis, seems promising. Unlike antibiotics, this approach is not target specific and it involves the modulation of various host protective responses and hence, is by and large impervious to the development of drug resistance. In the present study, CMN did not show direct inhibitory action on MTB but inhibited the survival of MTB strains inside the macrophages, indicating the mode of action by targeting of host responses which is yet to be discovered. Here, we do not wish to propose CMN as a therapeutic candidate but we are only putting forward a proof of concept. Hence, detailed investigation of the responses and mechanism underlying may lay a foundation for this novel approach having potential to be used as adjuvant to the existing drug regime.

\section{CONFLICTS OF INTEREST}

The authors confirm that this article content has no conflicts of interest.

\section{ACKNOWLEDGEMENT}

None declared.

\section{REFERENCES}

[1] World Health Organization. Global tuberculosis control report16. Geneva, WHO, 2011.

[2] Chadha VK. Tuberculosis epidemiology in India: a review. IJTLD 2005; 9: 1072-82.

[3] Masihi KN. Immunomodulators in infectious diseases: panoply of possibilities. Int J Immunopharm 2000; 22: 1083-91.

[4] Stanley SA, Raghavan S, Hwang WW, Cox JS. Acute infection and macrophage subversion by Mycobacterium tuberculosis require a specialized secretion system. Proc Natl Acad Sci USA 2003; 100: 13001-6.

[5] Srivastava RM, Singh S, Dubey SK, Misra K, Khar A. Immunomodulatory and therapeutic activity of curcumin. Int Immunopharm 2011; 11: 331-41.

[6] Changtam C, Hongmanee P, Suksamrarn A. Isoxazole analogs of curcuminoids with highly potent multidrug-resistant antimycobacterial activity. Eur J Med Chem 2010; 45: 4446-57.

[7] Canetti G, Fox W, Khomenko A, et al. Advances in techniques of testing mycobacterial drug sensitivity, and the use of sensitivity tests in tuberculosis control programmes. B World Health Organ $1969 ; 41: 21-43$.

[8] Palomino JC, Martin A, Camacho M, Guerra H, Swings J, Portaels F. Resazurin microtiter assay plate: simple and inexpensive method for detection of drug resistance in Mycobacterium tuberculosis. Antimicrob Agent Chemistries' 2002; 46: 2720-2.

[9] Hansen MB, Nielsen SE, Berg K. Re-examination and further development of a precise and rapid dye method for measuring cell growth/cell kill. J Immunological Methods 1989; 119: 203-10.

[10] Thierry C, Mary J, Hee KJ, et al. High content screening identifies decaprenyl-phosphoribose 2'epimerase as a target for intracellular antimycobacterial inhibitors. PLoS Pathog 2009; 5(10): e1000645. doi:10.1371/journal.ppat.1000645

[11] Wang JP, Hayashi T, Datta SK, Kornbluth RS, Raz E, Guiney DG. $\mathrm{CpG}$ oligonucleotides partially inhibit growth of Mycobacterium tuberculosis, but not Salmonella or Listeria, in human monocytederived macrophages. FEMS Immun Med Microbiol 2005; 45: 303-10.

[12] Hancock RE, Nijnik A, Philpott DJ. Modulating immunity as a therapy for bacterial infections. Nat Rev Microbiol 2012; 10: 24354. 
[13] Çıkrıkçı S, Mozioğlu E, Yılmaz H. Biological activity of curcuminoids isolated from Curcuma longa. Rec Nat Prod 2008; 2(1); 1924.

[14] Pampi C, Savita K, Ramakrishna R, Krishna S. Drug resistant clinical isolates of Mycobacterium tuberculosis from different genotypes exhibit differential host responses in THP-1 cells. PLoS One 2013; 8(5): e62966.
[15] Guo C, Rosoha E, Lowry MB, Borregaard N, Gombart AF. Curcumin induces human cathelicidin antimicrobial peptide gene expression through a vitamin D receptor-independent pathway. J Nutr Biochem 2012 (In Press)

[16] Sonawane A, Santos JC, Mishra BB, Jena P, Progida C, Sorensen $\mathrm{OE}$, et al. Cathelicidin is involved in the intracellular killing of mycobacteria in macrophages. Cell Microbiol 2011; 13: 1601-17.

Received: September 20,2013

Revised: October 20, 2013

Accepted: October 22, 2013

(c) Gupta et al.; Licensee Bentham Open.

This is an open access article licensed under the terms of the Creative Commons Attribution Non-Commercial License (http://creativecommons.org/licenses/ by-nc/3.0/) which permits unrestricted, non-commercial use, distribution and reproduction in any medium, provided the work is properly cited. 